\title{
Precision in Compositional Determination of Multiphase Nanoscale Structures Using the Aberration-corrected Advance Electron Microscope: Challenges and Opportunities
}

\author{
Khushubo Tiwari and Krishanu Biswas
}

Indian Institute of Technology Kanpur, Kanpur, Uttar Pradesh, India

Recently, the field of nanoalloys has attracted tremendous attention to the scientific community across various disciplines, due to our ability to tune properties of these materials by alloying as a function of composition, size, and shape [1]. Most importantly, chemistry plays a profound role in dictating phase evolution, interface structure and segregation behavior in nanoalloys [1, 2]. Hence the determination of the composition of these nanosized objects as precisely as possible is of paramount importance. Both EDX (energy-dispersive X-ray) and EELS (Electron energy-loss spectroscopy) are widely used to measure the composition of nanosized alloy particles. As compared to EELS, EDX is found to be versatile, easy-touse, fast and routine technique. Classically, EDX measurements are generally carried out employing the "Cliff-Lorimer" ratio method which requires the knowledge of the k-factors and their accurate determination is a fundamental point in order to precise quantification [3]. Though they can be evaluated experimentally or theoretically, the various limitation is observed (i) large uncertainties with k-factors in theoretical values (ii) the experimental k-factors requires many elements samples with well-known compositions and (iii) for light elements, prior information of samples mass thickness. X-ray absorption correction is important for low energy X-ray emissions. Therefore, to overcome these limitations, a new methodology named "Zeta $(\zeta)$ factors" method has been suggested [4]. In this method, to enabling X-ray absorption correction, the mass thickness and composition are calculated at the same time for each analysis point. In this regards several studies showed an excellent agreement between the expected results and theoretical values obtained using the zeta-factor method because their sample thickness is obtained for each analysis point.

In the present study, an attempt has been made to comprehend the challenges and complexities while performing the experiments using high-end microscopes. We have discussed some examples of the multiphase free and embedded nanoalloys based on our experimental studies. In these studies, we have considered both free and embedded nanoalloys in order to explore the elemental mapping of multiphase nanoscaled structure. For free nanoalloys, we have chosen bi-phasic $\mathrm{Pb}-17.5 \mathrm{at} \% \mathrm{Sb}$. These free nanoparticles (without matrix) were synthesized via the solvothermal route. Figure 1a shows the formation of bi-phasic alloy nanoparticle of $\mathrm{Pb}-\mathrm{Sb}$ by using high-angle annular dark-field imaging (HAADF) and EDX mapping [5]. Similarly, for embedded nanoparticle, tri-phasic Bi44In32 $\mathrm{Sn}_{23}$ alloy nanoparticle embedded in icosahedral quasicrystalline (A165Cu20Fe15) matrix was prepared by rapid solidification (melt spinning) route. Figure $1 \mathrm{~b}$ shows the three-phase contrast in the HAADF image, clearly revealing the formation of three distinct phases of $(\mathrm{Bi})$, Biln and $(\gamma-\mathrm{Sn})$. Also, their elemental maps are used to ascertain the presence of constituent elements. Our samples were measured using a $300 \mathrm{kV}$ aberrationcorrected TITAN (FEI, The Netherlands) and equipped with an advance QUANTAX EDS features the XFlash with 6T detector with a nanobeam spot size of $1.5 \pm 0.5 \mathrm{~nm}$. As it is well known that free nanoalloys offer opportunities to understand the phase transformation behavior as they do not comprise of any matrix. Therefore, in recent times, the free nanoalloys have gained a lot of attention due to the potential opportunity of studying the inherent size and shape-dependent behavior of nanoparticles. But synthesis is another significant challenge to the research community. These advanced methods of chemical quantification somehow help us to acquire an exact measurements for free nanoalloys. Though free 
nanoalloy particles are in contact with the amorphous carbon film while performing TEM investigation, it is anticipated that the existence of few tens of nm thick carbon film at the nanoparticle surface does not alter significantly while working in the experimental condition. It has been noticed that the $\zeta$ - factors approach is perfectly fit for thin samples but gets difficulty for thicker specimens, where fluorescence and absorbance become important factors. Likewise for embedded nanoalloy, decoupling of the effect of the matrix in the nanoparticle itself a challenge, so this method could not be applied to this condition as nanoalloy particle is always associated with the layer of matrix surface. Particularly in these kinds of samples, they contribute to inaccurate quantification of the composition of nanoalloy particles in interest when it is stacked by layers of matrix surface and particles. Thus, in our opinion the best way to obtain approximate data is to prepare a wedge-thinned sample by FIB (focused ion beam) milling because on the basis of sample geometry, the local thickness can be precisely determined and further this allows to obtain the factor by the extrapolation method. Other limitations while performing experiments can be handled by (i) minimizing contamination by keeping sample temperature less than $100 \mathrm{~K}$ during the analysis by utilizing liquid nitrogen cooled double tilt holder (ii) the drifting can be reduced by using the anti-drift correction. Now, these tools are easily available in the FEI, TIA software. To obtain good maps quality of the sample, the longer acquisition times allow better structure as compare to small acquisition time. Hence optimizations of these parameters are expected to yield precise determination of chemistry of nanoalloys.
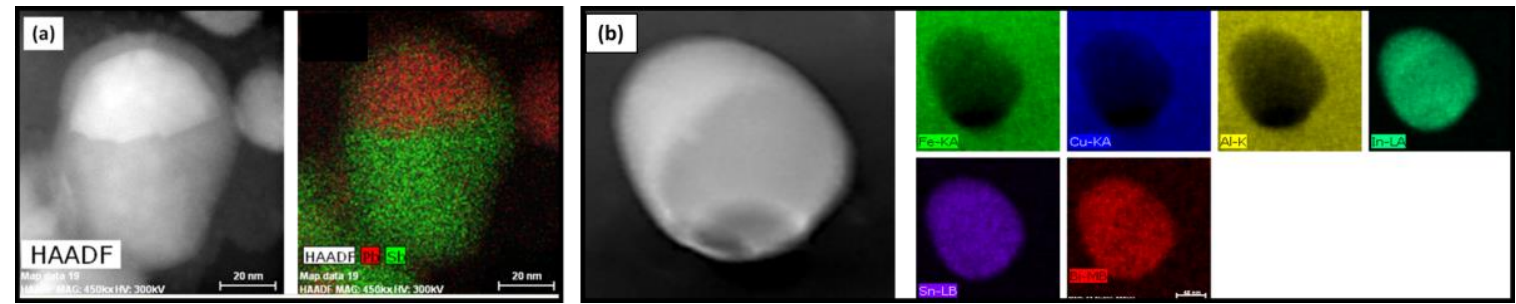

Figure 1. HAADF-STEM-EDS elemental map showing the distribution of (a) $\mathrm{Pb}$ and $\mathrm{Sb}$ in bi-phasic $\mathrm{Pb}-$ $17.5 \mathrm{at} \% \mathrm{Sb}$ [5] and (b) Bi, In Sn, Al, Cu and Fe in tri-phasic Bi44In32Sn23 alloy nanoparticle embedded in icosahedral quasicrystalline matrix.

References

[1] F. Calvo, Phys Chem Chem Phys, 17 (2015), p. 27922.

[2] K. Chattopadhyay, T Indian I Metals, 58 (2005), p. 1057.

[3] G. Cliff and G. W. Lorimer, J. Microsc., 203 (1975), p. 103.

[4] M. Watanabe and D.B. Williams, J. Microsc., 221 (2006), p. 89

[5] M. M. Devi, K. Tiwari, and K. Biswas, Metall. Mater. Trans., 50 (2019), p. 3959. 